\title{
Integer Polynomial Optimization in Fixed Dimension
}

\author{
Jesús A. De Loera \\ University of California, Dept. of Mathematics, Davis CA 95616, USA \\ email: deloera@math.ucdavis.edu http://www.math.ucdavis.edu/ deloera
}

Raymond Hemmecke

Otto-von-Guericke-Universität Magdeburg, FMA/IMO, Universitätsplatz 2, 39106 Magdeburg, Germany email: hemmecke@imo.math.uni-magdeburg.de http://www.math.uni-magdeburg.de/ ${ }^{\sim h e m m e c k e}$

Matthias Köppe

Otto-von-Guericke-Universität Magdeburg, FMA/IMO, Universitätsplatz 2, 39106 Magdeburg, Germany email: mkoeppe@imo.math.uni-magdeburg.de http://www.math.uni-magdeburg.de/〜mkoeppe

Robert Weismantel

Otto-von-Guericke-Universität Magdeburg, FMA/IMO, Universitätsplatz 2, 39106 Magdeburg, Germany email: weismant@imo.math.uni-magdeburg.de http://www.math.uni-magdeburg.de/〜weismant

We classify, according to their computational complexity, integer optimization problems whose constraints and objective functions are polynomials with integer coefficients and the number of variables is fixed. For the optimization of an integer polynomial over the lattice points of a convex polytope, we show an algorithm to compute lower and upper bounds for the optimal value. For polynomials that are non-negative over the polytope, these sequences of bounds lead to a fully polynomial-time approximation scheme for the optimization problem.

Key words: integer non-linear programming, integer programming in fixed dimension, computational complexity, rational functions, approximation algorithms, FPTAS.

MSC2000 Subject Classification: Primary: 90C10, 90C30; Secondary: 90C60, 90C57

OR/MS subject classification: Primary: Programming, Integer, Nonlinear; Secondary: Analysis of algorithms, Computational complexity

1. Introduction Mixed integer non-linear programs combine the hardness of combinatorial explosion with the non-convexity of non-linear functions. For example, the well-known optimality conditions developed for differentiable objective functions have no meaning when the variables are discrete. Thus, it is perhaps not surprising that already linear integer programming with general quadratic constraints is undecidable 10. Nevertheless, when the number of variables is fixed discrete optimization problems often become tractable and efficient polynomial algorithms exist (e.g. 1, 9, 14 ). It is thus natural to ask what is the complexity of integer non-linear optimization assuming that the number of variables is fixed? We study the problem

$$
\text { maximize } f\left(x_{1}, \ldots, x_{d}\right) \text { subject to } g_{i}\left(x_{1}, \ldots, x_{d}\right) \geq 0, x \in \mathbb{Z}^{d} .
$$

Here $f, g_{i}$ are polynomials with integral coefficients. Note that all throughout the paper we assume that the number of variables is fixed. Here are our two contributions to the theory:

(1) We give a classification of the computational complexity of Problem (1) according to special cases. Section 2 of this article presents the details, but the reader can see the classification in Table 1 New results are marked with letters, known results are marked with asterisks, arrows indicate implications:

Table 1: Computational complexity of problem (1) in fixed dimension.

\begin{tabular}{|c|c|c|c|}
\hline \multirow[b]{2}{*}{ Type of constraints } & \multicolumn{3}{|c|}{ Type of objective function } \\
\hline & linear & $\begin{array}{c}\text { convex } \\
\text { polynomial }\end{array}$ & $\begin{array}{c}\text { arbitrary } \\
\text { polynomial }\end{array}$ \\
\hline Linear constraints, integer variables & $\underset{\Uparrow}{\operatorname{polytime}}(*)$ & $\Leftarrow \underset{\uparrow}{\operatorname{polytime}}(* *)$ & NP-hard (a) \\
\hline Convex semialgebraic constraints, integer variables & polytime $(* *)$ & $\Leftarrow$ polytime $(* *)$ & NP-hard (c) \\
\hline Arbitrary polynomial constraints, integer variables & \multicolumn{3}{|c|}{ undecidable $(\mathrm{b}) \Rightarrow$ undecidable $(\mathrm{d}) \Rightarrow$ undecidable $(\mathrm{e})$} \\
\hline
\end{tabular}

(2) For problem (a), that of optimizing an arbitrary integral polynomial over the lattice points of a convex 
rational polytope with fixed number of variables, we present an algorithm to compute a sequence of upper and lower bounds for its optimal value. Our bounds can be used, for instance, in a branch-and-bound search for the optimum. We use Barvinok's algebraic encoding of the lattice points of polytopes via rational functions [2]. In Section 3 we prove:

THEOREM 1.1 Let the number of variables $d$ be fixed. Let $f\left(x_{1}, \ldots, x_{d}\right)$ be a polynomial of maximum total degree $D$ with integer coefficients, and let $P$ be a convex rational polytope defined by linear inequalities in $d$ variables. We obtain an increasing sequence of lower bounds $\left\{L_{k}\right\}$ and a decreasing sequence of upper bounds $\left\{U_{k}\right\}$ to the optimal value

$$
f^{*}=\text { maximize } f\left(x_{1}, x_{2}, \ldots, x_{d}\right) \text { subject to } x \in P \cap \mathbb{Z}^{d} .
$$

The bounds $L_{k}, U_{k}$ can be computed in time polynomial in $k$, the input size of $P$ and $f$, and the maximum total degree $D$ and they satisfy the inequality $U_{k}-L_{k} \leq f^{*} \cdot\left(\sqrt[k]{\left|P \cap \mathbb{Z}^{d}\right|}-1\right)$.

More strongly, if $f$ is non-negative over the polytope (i.e. $f(x) \geq 0$ for all $x \in P$ ), there exists a fully polynomial-time approximation scheme (FPTAS) for the optimization problem (2).

We conclude with examples and a brief look at the mixed integer problem.

2. Computational Complexity Bounds All the results we present refer to the complexity model where the number of operations is given in terms of the input size measured in the standard binary encoding. The results of H. W. Lenstra Jr. [14 imply the entry of Table 1 marked with (*), i.e. solving linear integer programming problems with a fixed number of variables can be done in time polynomial in the size of the input. More recently, Khachiyan and Porkolab [9] have proved that in fixed dimension, the problem of minimizing a convex polynomial objective function over the integers, subject to polynomial constraints that define a convex body, can be solved in polynomial time in the encoding length of the input. Thus, they settled all entries marked by $(* *)$. By the natural containment exhibited by these complexity classes, to show the validity of the remaining entries of Table 1 is enough to prove the following lemma:

LEMma $2.1 \quad$ (i) The problem of minimizing a degree four polynomial over the lattice points of a convex polygon is NP-hard (entry (a) in Table 1).

(ii) The problem of minimizing a linear form over polynomial constraints in at most 10 integer variables is not computable by a recursive function (entry (b) in Table 1).

Proof. (1) We use the NP-complete problem AN1 on page 249 of [7. This problem states it is NP-complete to decide whether, given three positive integers $a, b, c$, there exists a positive integer $x<c$ such that $x^{2}$ is congruent with $a$ modulo $b$. This problem is clearly equivalent to asking whether the minimum of the quartic polynomial function $\left(x^{2}-a-b y\right)^{2}$ over the lattice points of the rectangle $\left\{(x, y) \mid 1 \leq x \leq c-1, \frac{1-a}{b} \leq y \leq \frac{(c-1)^{2}-a}{b}\right\}$ is zero or not. This settles part (1).

(2) In 1973 Jeroslow 10 proved a similar result without fixing the number of variables. We follow his idea, but resorting to a stronger lemma. More precisely our proof relies on a 1982 result 11 which states that there is no recursive function that, given an integer polynomial $f$ with nine variables, can determine whether $f$ has a non-negative integer zero, in the sense that it finds an explicit zero or returns null otherwise. Jones paper is a strengthening of the original solution of Hilbert's tenth problem [15]. Now to each polynomial $f$ in $\mathbb{Z}\left[x_{1}, x_{2} \ldots, x_{9}\right]$ associate the ten-dimensional minimization problem

$$
\text { minimize } y \text { subject to }(1-y) f\left(x_{1}, x_{2}, \ldots, x_{9}\right)=0,\left(y, x_{1}, \ldots, x_{9}\right) \in \mathbb{Z}_{\geq 0}^{10} \text {. }
$$

The minimum attained by $y$ is either zero or one depending on whether $f$ has an integer non-negative solution or not. Thus part (2) is settled.

3. FPTAS for Optimizing Non-Negative Polynomials over Integer Points of Polytopes Consider now a polynomial function $f \in \mathbb{Z}\left[x_{1}, x_{2}, \ldots, x_{d}\right]$ of maximum total degree $D$ and a convex polytope $P=\{x \mid A x \leq b\}$ where $A$ is an $m \times d$ integral matrix and $b$ is an integral $m$-vector. The purpose of this section is to present an algorithm to generate lower and upper bounds $L_{k}, U_{k}$ to the integer global optimum value of

$$
\text { maximize } f\left(x_{1}, \ldots, x_{d}\right) \text { subject to }\left(x_{1}, \ldots, x_{d}\right) \in P \cap \mathbb{Z}^{d} \text {. }
$$


We should also remark that in our algorithm the polynomial objective function $f$ can be arbitrary (e.g. non-convex). As we have seen, the optimization problem is NP-hard already for two integer variables and polynomials of degree four. Nevertheless we will see that, in fixed dimension and when $f(x) \geq 0$ for all $x \in P$, the algorithm gives a fully polynomial time approximation scheme or FPTAS. This means that, in polynomial time on the input and $(1 / \epsilon)$, one can compute a $(1-\epsilon)$-approximation to the maximum. The algorithm we present is based on A. Barvinok's theory for encoding all the lattice points of a polyhedron in terms of short rational functions. See [1, 2] for all details. Lattice points are thought of as exponent vectors of monomials. For example, $z_{1}^{2} z_{2}^{-11}$ encodes the lattice point $(2,-11)$. The set of lattice points is represented by a Laurent polynomial: $g_{P}(z)=\sum_{\alpha \in P \cap \mathbb{Z}^{d}} z^{\alpha}$. From Barvinok's theory this exponentiallylarge sum of monomials $g_{P}(z)$ can instead be written as a polynomial-size sum of rational functions (assuming the dimension $d$ is fixed) of the form:

$$
g_{P}(z)=\sum_{i \in I} E_{i} \frac{z^{u_{i}}}{\prod_{j=1}^{d}\left(1-z^{v_{i j}}\right)},
$$

where $I$ is a polynomial-size indexing set, and where $E_{i} \in\{1,-1\}$ and $u_{i}, v_{i j} \in \mathbb{Z}^{d}$ for all $i$ and $j$. For details see [2, 5, 6].

We need a way to encode via rational functions the values of the polynomial $f$ over all the lattice points in a polytope. The key idea, first introduced in Lemma 9 of [6] and generalized in [8], is that differential operators associated to $f$ can be used to compute a rational function representation of $\sum_{a \in P \cap \mathbb{Z}^{d}} f(a) z^{a}$. The following Lemma recently appeared in [3]:

LEMma 3.1 Let $g_{P}(z)$ be the Barvinok representation of the generating function of the lattice points of $P$. Let $f$ be a polynomial in $\mathbb{Z}\left[x_{1}, \ldots, x_{d}\right]$ of maximum total degree $D$. We can compute, in time polynomial on $D$ and the size of the input data, a Barvinok rational function representation $g_{P, f}(z)$ for the generating function $\sum_{a \in P \cap \mathbb{Z}^{d}} f(a) z^{a}$.

Proof. We give here the author's original proof the lemma for $D$ fixed. The first proof without this assumption was recently given by A. Barvinok in $[3]$.

We begin assuming $f(z)=z_{r}$, the general case will follow from it: Consider the action of the differential operator $z_{r} \frac{\partial}{\partial z_{r}}$ in the generating function $g_{P}(z)$ and on its Barvinok representation. On one hand, for the generating function

$$
z_{r} \frac{\partial}{\partial z_{r}} \cdot g_{P}(z)=\sum_{\alpha \in P \cap \mathbb{Z}^{d}} z_{r} \frac{\partial}{\partial z_{r}} z^{\alpha}=\sum_{\alpha \in P \cap \mathbb{Z}^{d}} \alpha_{r} z^{\alpha} .
$$

On the other hand, by linearity of the operator, we have that in terms of rational functions

$$
z_{r} \frac{\partial}{\partial z_{r}} \cdot g_{P}(z)=\sum_{i \in I} E_{i} z_{r} \frac{\partial}{\partial z_{r}} \cdot\left(\frac{z^{u_{i}}}{\prod_{j=1}^{d}\left(1-z^{v_{i j}}\right)}\right) .
$$

Thus it is enough to prove that the summands of the expression above can be written in terms of rational functions computable in polynomial time. The standard quotient rule for derivatives says that

$$
\frac{\partial}{\partial z_{r}}\left(\frac{z^{u_{i}}}{\prod_{j=1}^{d}\left(1-z^{v_{i j}}\right)}\right)=\frac{\left(\frac{\partial z^{u_{i}}}{\partial z_{r}}\right) \prod_{j=1}^{d}\left(1-z^{v_{i j}}\right)-z^{u_{i}}\left(\frac{\partial}{\partial z_{r}} \prod_{j=1}^{d}\left(1-z^{v_{i j}}\right)\right)}{\prod_{j=1}^{d}\left(1-z^{v_{i j}}\right)^{2}} .
$$

We can expand the numerator as a sum of no more than $2^{d}$ monomials. This is a constant number because $d$, the number of variables, is assumed to be a constant. This argument completes the proof of our lemma when $f(z)=z_{r}$.

For the case when $f(z)$ is a general monomial, i.e. $f(z)=c \cdot z_{1}^{\beta_{1}} \cdot \ldots \cdot z_{d}^{\beta_{d}}$, then we can compute again a rational function representation of $g_{P, f}(z)$ by repeated application of basic differential operators:

$$
c\left(z_{1} \frac{\partial}{\partial z_{1}}\right)^{\beta_{1}} \cdot \ldots \cdot\left(z_{d} \frac{\partial}{\partial z_{d}}\right)^{\beta_{d}} \cdot g_{P}(z)=\sum_{\alpha \in P \cap \mathbb{Z}^{d}} c \cdot \alpha^{\beta} z^{\alpha} .
$$


Thus we require no more than $O\left(D^{d}\right)$ repetitions of the single-variable case.

Finally, if we deal with a polynomial $f$ of many monomial terms, we compute and add up all such expressions that we get for each term of $f(x)$ and obtain our desired short rational function representation for the generating function for $\sum_{\alpha \in P \cap \mathbb{Z}^{d}} f(\alpha) z^{\alpha}$. Note that only polynomially many steps are needed because $d$ is fixed and the largest number of possible monomials in $f$ of degree $s$ is $\left(\begin{array}{c}d+s-1 \\ d-1\end{array}\right)$, thus for fixed $d$ we will do no more than $O\left(D^{d}\right)$ repetitions of the monomial case.

Now we are ready to present our algorithm to obtain bounds $U_{k}, L_{k}$ that reach the optimum. Step 1 of preprocessing is necessary because we rely on the elementary fact that, for a collection $S=\left\{s_{1}, \ldots, s_{r}\right\}$ of non-negative real numbers, maximum $\left\{s_{i} \mid s_{i} \in S\right\}$ equals $\lim _{k \rightarrow \infty} \sqrt[k]{\sum_{j=1}^{r} s_{j}^{k}}$.

\section{Algorithm}

Input: A rational convex polytope $P \subset \mathbb{R}^{d}$, a polynomial objective $f \in \mathbb{Z}\left[x_{1}, \ldots, x_{d}\right]$ of maximum total degree $D$.

Output: An increasing sequence of lower bounds $L_{k}$, and a decreasing sequence of upper bounds $U_{k}$ reaching the maximal function value $f^{*}$ of $f$ over all lattice points of $P$.

Step 1. If $f$ is known to be non-negative in all points of $P$, then go directly to Step 2 . Else, solving $2 d$ linear programs over $P$, we find lower and upper integer bounds for each of the variables $x_{1}, \ldots, x_{d}$. Let $M$ be the maximum of the absolute values of these $2 d$ numbers. Thus $\left|x_{i}\right| \leq M$ for all $i$. Let $C$ be the maximum of the absolute values of all coefficients, and $r$ be the number of monomials of $f(x)$. Then

$$
L:=-r C M^{D} \leq f(x) \leq r C M^{D}=: U,
$$

as we can bound the absolute value of each monomial of $f(x)$ by $C M^{D}$. Replace $f$ by $\bar{f}(x)=f(x)-L \leq$ $U-L$, a non-negative polynomial over $P$. Go to Steps 2,3 , etc. and return the optimal value of $\overline{\bar{f}}$. Trivially, if we find the optimal value of $\bar{f}$ over $P$ we can extract the optimal value for $f$.

Step 2. Via Barvinok's algorithm (see [1, 2] 3]), compute a short rational function expression for the generating function $g_{P}(z)=\sum_{\alpha \in P \cap \mathbb{Z}^{d}} z^{\alpha}$. From $g_{P}(z)$ compute the number $\left|P \cap \mathbb{Z}^{d}\right|=g_{P}(1)$ of lattice points in $P$ in polynomial time.

Step 3. From the rational function representation $g_{P}(z)$ of the generating function $\sum_{\alpha \in P \cap \mathbb{Z}^{d}} z^{\alpha}$ compute the rational function representation of $g_{P, f^{k}}(z)$ of $\sum_{\alpha \in P \cap \mathbb{Z}^{d}} f^{k}(\alpha) z^{\alpha}$ in polynomial time by application of Lemma 3.1. We define

$$
L_{k}:=\sqrt[k]{g_{P, f^{k}}(1) / g_{P, f^{0}}(1)} \text { and } U_{k}:=\sqrt[k]{g_{P, f^{k}}(1)}
$$

When $\left\lfloor U_{k}\right\rfloor-\left\lceil L_{k}\right\rceil<1$ stop and return $\left\lceil L_{k}\right\rceil=\left\lfloor U_{k}\right\rfloor$ as the optimal value.

\section{End of Algorithm.}

\section{LEMMA 3.2 The algorithm is correct.}

ProOF. Using the fact that the arithmetic mean of a finite set of nonnegative values is at most as big as the maximum value, which in turn is at most as big as the sum of all values, we obtain the sequences of lower and upper bounds, $L_{k}$ and $U_{k}$, for the maximum:

$$
L_{k}=\sqrt[k]{\frac{\sum_{\alpha \in P \cap \mathbb{Z}^{d}} f(\alpha)^{k}}{\left|P \cap \mathbb{Z}^{d}\right|}} \leq \max \left\{f(\alpha): \alpha \in P \cap \mathbb{Z}^{d}\right\} \leq \sqrt[k]{\sum_{\alpha \in P \cap \mathbb{Z}^{d}} f(\alpha)^{k}}=U_{k} .
$$

Note that as $s \rightarrow \infty, L_{k}$ and $U_{k}$ approach this maximum value monotonously (from below and above, respectively). Trivially, if the difference between (rounded) upper and lower bounds becomes strictly less than 1 , we have determined the value $\max \left\{f(x): x \in P \cap \mathbb{Z}^{d}\right\}=\left\lceil L_{k}\right\rceil$. Thus the algorithm terminates with the correct answer.

Theorem 1.1 will follow from the next lemma: 
Lemma 3.3 Let $f$ be a polynomial with integer coefficients and maximum total degree D. When the dimension $d$ is fixed,

(i) the bounds $L_{k}, U_{k}$ can be computed in time polynomial in $k$, the input size of $P$ and $f$, and the total degree $D$. The bounds satisfy the following inequality:

$$
U_{k}-L_{k} \leq f^{*} \cdot\left(\sqrt[k]{\left|P \cap \mathbb{Z}^{d}\right|}-1\right)
$$

(ii) In addition, when $f$ is non-negative over $P$ (i.e. $f(x) \geq 0$ for all $x \in P)$, for $k=(1+$ $1 / \epsilon) \log \left(\left|P \cap \mathbb{Z}^{d}\right|\right), L_{k}$ is a $(1-\epsilon)$-approximation to the optimal value $f^{*}$ and it can be computed in time polynomial in the input size, the total degree $D$, and $1 / \epsilon$. Similarly, $U_{k}$ gives a $(1+\epsilon)$ approximation to $f^{*}$. Moreover, with the same complexity, one can also find a feasible lattice point that approximates an optimal solution with similar quality.

Proof. Part (i). From Lemma 3.1 on fixed dimension $d$, we can compute $g_{P, f}=\sum_{\alpha \in P \cap \mathbb{Z}^{d}} f(\alpha) z^{\alpha}$ as a rational function in time polynomial in $D$, the total degree of $f$, and the input size of $P$. Thus, because $f^{k}$ has total degree of $D k$ and the encoding length for the coefficients of $f^{k}$ is bounded by $k \log (k C)$ (with $C$ the largest coefficient in $f$ ), we can also compute $g_{P, f^{k}}=\sum_{\alpha \in P \cap \mathbb{Z}^{d}} f^{k}(\alpha) z^{\alpha}$ in time polynomial in $k$, the total degree $D$, and the input size of $P$. Note that using residue techniques [3], we can evaluate $g_{P, f^{k}}(1)$ in polynomial time. Finally observe

$$
\begin{aligned}
U_{k}-L_{k} & =\sqrt[k]{\sum_{\alpha \in P \cap \mathbb{Z}^{d}} f^{k}(\alpha)}-\sqrt[k]{\frac{\sum_{\alpha \in P \cap \mathbb{Z}^{d}} f^{k}(\alpha)}{\left|P \cap \mathbb{Z}^{d}\right|}}=\sqrt[k]{\frac{\sum_{\alpha \in P \cap \mathbb{Z}^{d}} f^{k}(\alpha)}{\left|P \cap \mathbb{Z}^{d}\right|}}\left(\sqrt[k]{\left|P \cap \mathbb{Z}^{d}\right|}-1\right) \\
& =L_{k}\left(\sqrt[k]{\left|P \cap \mathbb{Z}^{d}\right|}-1\right) \leq f^{*}\left(\sqrt[k]{\left|P \cap \mathbb{Z}^{d}\right|}-1\right)
\end{aligned}
$$

Part (ii). Note that if $\left(\sqrt[k]{\left|P \cap \mathbb{Z}^{d}\right|}-1\right) \leq \epsilon$ then $L_{k}$ is indeed a $(1-\epsilon)$-approximation because

$$
f^{*} \leq U_{k}=L_{k}+\left(U_{k}-L_{k}\right) \leq L_{k}+f^{*}\left(\sqrt[k]{\left|P \cap \mathbb{Z}^{d}\right|}-1\right) \leq L_{k}+f^{*} \epsilon
$$

Observe that $\phi(\epsilon):=(1+1 / \epsilon) /(1 / \log (1+\epsilon))$ is an increasing function for $\epsilon<1$ and $\lim _{\epsilon \rightarrow 0} \phi(\epsilon)=1$, thus $\phi(\epsilon) \geq 1$ for $0<\epsilon \leq 1$. Hence, for all $k \geq \log \left(\left|P \cap \mathbb{Z}^{d}\right|\right)+\log \left(\left|P \cap \mathbb{Z}^{d}\right|\right) / \epsilon \geq \log \left(\left|P \cap \mathbb{Z}^{d}\right|\right) / \log (1+$ $\epsilon)$, we have indeed $\left(\sqrt[k]{\left|P \cap \mathbb{Z}^{d}\right|}-1\right) \leq \epsilon$. Finally, from Lemma 3.1 the calculation of $L_{k}$ for $k=$ $\log \left(\left|P \cap \mathbb{Z}^{d}\right|\right)+\log \left(\left|P \cap \mathbb{Z}^{d}\right|\right) / \epsilon$ would require a number of steps polynomial in the input size and $1 / \epsilon$. A very similar argument can be written for $U_{k}$ but we omit it here.

To complete the proof of part (ii) it remains to show that not only we approximate the optimal value $f^{*}$ but we can also efficiently find a lattice point $\alpha$ with $f(\alpha)$ giving that quality approximation of $f^{*}$. Let $k=(1+1 / \epsilon) \log \left(\left|P \cap \mathbb{Z}^{d}\right|\right)$, thus, by the above discussion, $L_{k}$ is an $(1-\epsilon)$-approximation to $f^{*}$. Let $Q_{0}:=[-M, M]^{d}$ denote the box computed in Step 1 of the algorithm such that $P \subseteq Q_{0}$. By bisecting $Q_{0}$, we obtain two boxes $Q_{1}^{\prime}$ and $Q_{1}^{\prime \prime}$. By applying the algorithm separately to the polyhedra $P \cap Q_{1}^{\prime}$ and $P \cap Q_{1}^{\prime \prime}$, we compute lower bounds $L_{k}^{\prime}$ and $L_{k}^{\prime \prime}$ for the optimization problems restricted to $Q_{1}^{\prime}$ and $Q_{1}^{\prime \prime}$, respectively. Because $L_{k}^{k}$ is the arithmetic mean of $f^{k}(\alpha)$ for $\alpha \in P \cap \mathbb{Z}^{d}$, clearly

$$
\min \left\{L_{k}^{\prime}, L_{k}^{\prime \prime}\right\} \leq L_{k} \leq \max \left\{L_{k}^{\prime}, L_{k}^{\prime \prime}\right\} .
$$

Without loss of generality, let $L_{k}^{\prime} \geq L_{k}^{\prime \prime}$. We now apply the bisection procedure iteratively on $Q_{k}^{\prime}$. After $d \log M$ bisection steps, we obtain a box $Q_{k}^{\prime}$ that contains a single lattice point $\alpha \in P \cap Q_{k}^{\prime} \cap Z^{d}$, which has an objective value $f(\alpha)=L_{k}^{\prime} \geq L_{k} \geq(1-\epsilon) f^{*}$.

We remark that if we need to apply the construction of Step 1 of the algorithm because $f$ takes negative values on $P$, then we can only obtain an $(1-\epsilon)$-approximation (and $(1+\epsilon)$-approximation, respectively) for the modified function $\bar{f}$ in polynomial time, but not the original function $f$. We also emphasize that, although our algorithm requires the computation of $\sum_{\alpha \in P} f^{q}(\alpha)$ for different powers of $f$, these numbers are obtained without explicitly listing all lattice points (a hard task), nor we assume any knowledge of the individual values $f(\alpha)$. We can access the power means $\sum_{\alpha \in P} f^{q}(\alpha)$ indirectly via rational functions. Here are two small examples: 
Example 1, monomial optimization over a quadrilateral: The problem we consider is that of maximizing the value of the monomial $x^{3} y$ over the lattice points of the quadrilateral

$$
\{(x, y) \mid 3991 \leq 3996 x-4 y \leq 3993,1 / 2 \leq x \leq 5 / 2\} .
$$

It contains only 2 lattice points. The sum of rational functions encoding the lattice points is

$$
\frac{x^{2} y^{1000}}{\left(1-\left(x y^{999}\right)^{-1}\right)\left(1-y^{-1}\right)}+\frac{x y}{\left(1-x y^{999}\right)\left(1-y^{-1}\right)}+\frac{x y}{\left(1-x y^{999}\right)(1-y)}+\frac{x^{2} y^{1000}}{\left(1-\left(x y^{999}\right)^{-1}\right)(1-y)} \text {. }
$$

In the first iteration $L_{1}=4000.50$ while $U_{1}=8001$. After thirty iterations, we see $L_{30}=7817.279750$ while $U_{30}=8000$, the true optimal value.

Example 2, nvs04 from MINLPLIB: A somewhat more complicated example, from a well-known library of test examples (see http://www.gamsworld.org/minlp/), is the problem given by

$$
\begin{array}{ll}
\min & 100\left(\frac{1}{2}+i_{2}-\left(\frac{3}{5}+i_{1}\right)^{2}\right)^{2}+\left(\frac{2}{5}-i_{1}\right)^{2} \\
\text { s.t. } & i_{1}, i_{2} \in[0,200] \cap \mathbb{Z} .
\end{array}
$$

Its optimal solution as given in MINLPLIB is $i_{1}=1, i_{2}=2$ with an objective value of 0.72 . Clearly, to apply our algorithm from page 4 literally, the objective function needs to be multiplied by a factor of 100 to obtain an integer valued polynomial.

Using the bounds on $i_{1}$ and $i_{2}$ we obtain an upper bound of $165 \cdot 10^{9}$ for the objective function, which allows us to convert the problem into an equivalent maximization problem, where all feasible points have a non-negative objective value. The new optimal objective value is 164999999999.28. Expanding the new objective function and translating it into a differential operator yields

$$
\begin{aligned}
& \frac{4124999999947}{25} \operatorname{Id}-28 z_{2} \frac{\partial}{\partial z_{2}}+\frac{172}{5} z_{1} \frac{\partial}{\partial z_{1}}-117\left(z_{1} \frac{\partial}{\partial z_{1}}\right)^{(2)}-100\left(z_{2} \frac{\partial}{\partial z_{2}}\right)^{(2)} \\
& +240\left(z_{2} \frac{\partial}{\partial z_{2}}\right)\left(z_{1} \frac{\partial}{\partial z_{1}}\right)+200\left(z_{2} \frac{\partial}{\partial z_{2}}\right)\left(z_{1} \frac{\partial}{\partial z_{1}}\right)^{(2)}-240\left(z_{1} \frac{\partial}{\partial z_{1}}\right)^{(3)}-100\left(z_{1} \frac{\partial}{\partial z_{1}}\right)^{(4)} .
\end{aligned}
$$

The short generating function can be written as $g\left(z_{1}, z_{2}\right)=\left(\frac{1}{1-z_{1}}-\frac{z_{1}^{201}}{1-z_{1}}\right)\left(\frac{1}{1-z_{2}}-\frac{z_{2}^{201}}{1-z_{2}}\right)$.

In this example, the number of lattice points is $\left|P \cap \mathbb{Z}^{2}\right|=40401$. The first bounds are $L_{1}=$ 139463892042.292155534, $U_{1}=28032242300500.723262442$. After 30 iterations the bounds become $L_{30}=164999998845.993553019$ and $U_{30}=165000000475.892451381$.

4. An extension to the mixed integer case Now, we wish to discuss extensions of Theorem 1.1 to the mixed integer scenario. If some of the variables are allowed to be continuous then we can describe the task as

$$
\text { maximize } f(x, y) \text { subject to }\left\{(x, y) \mid A x+B y \leq b \text {, with } x_{i} \in \mathbb{Z} \text { and } y_{i} \in \mathbb{R}\right\} .
$$

Consider the sequence of integer polynomial optimization problems optimize $f\left(x, \frac{y}{n}\right)$ subject to $(x, y) \in$ $\Gamma_{n}=\left\{(x, y) \mid A x+B\left(\frac{y}{n}\right) \leq b\right.$, with $x_{i} \in \mathbb{Z}$ and $\left.y_{i} \in \mathbb{Z}\right\}$, where each of the subproblems is equivalent to optimizing a polynomial over a "semi-dilated" polytope (in some coordinate directions but not others). As $n$ goes to infinity, the sequence of optimal solution values can have several limit points. Nevertheless, it is still possible to construct a subsequence of problems whose optimal values approximate the mixed integer optimum to arbitrary precision:

COROLLARY 4.1 With the hypotheses of Theorem 1.1. we can construct a sequence of integer polynomial programming problems, over finer and finer grids, whose optimal values converge to the optimal value of the mixed integer program

$$
f^{*}=\text { maximize } f\left(x_{1}, x_{2}, \ldots, x_{d}\right) \text { subject to } x \in P, \text { and } x_{i} \in \mathbb{Z} \text { for } i \in I \subseteq\{1,2, \ldots, d\} .
$$

By applying the algorithm of Theorem 1.1 to the subproblems, we can approximate the optimum to arbitrary precision. 
When all variables are continuous, the original polytope $P$ is actually dilated uniformly in all directions by a parameter $n$, or equivalently, the integer grid is refined. Define

$$
r(q, n)=\sum_{\alpha \in(n P) \cap \mathbb{Z}^{d}} f^{q}(\alpha) z^{\alpha},
$$

for each power $q$ and dilation factor $n$. Note that for fixed $q$, then we can easily see that the sum $\sum_{\alpha \in(n P) \cap \mathbb{Z}^{d}} \frac{1}{n^{d}} f^{q}(\alpha) z^{\alpha}$ is essentially an approximation to the Riemann integral of $f^{q}$; thus

$$
\lim _{n \rightarrow \infty} r(q, n) / n^{d}=\int_{P} f^{q}(\bar{x}) d \bar{x} .
$$

As $n, q$ grow, the values $\sqrt[q]{r(q, n)}$ approximate the sequence $\sqrt[q]{\int_{P} f^{q}(\bar{x}) d \bar{x}}$ which converges to $\left(\max _{x \in P} f(x)\right) \cdot \operatorname{volume}(P)$. This is related to recent work (see [4, 13, 16] and references therein) where the global optimum of a polynomial over a compact domain is investigated as the result of a grid refinement and properties of sums of squares.

Acknowledgments. We are truly grateful to Prof. Alexander Barvinok who communicated to us that Lemma 3.1 was true for variable $D$ and thus we had indeed obtained an FPTAS from the construction of the upper and lower bounds. We thank the anonymous referees for the many suggestions that improved the presentation. The first author gratefully acknowledges support from NSF grant DMS-0309694, a 2003 UC-Davis Chancellor's fellow award, the Alexander von Humboldt foundation, and IMO-Magdeburg. The remaining authors were supported by the European TMR network ADONET 504438.

\section{References}

[1] Barvinok, A.I. Polynomial time algorithm for counting integral points in polyhedra when the dimension is fixed. Math of Operations Research 19, 1994, 769-779.

[2] Barvinok, A.I. and Pommersheim, J. An algorithmic theory of lattice points in polyhedra. In: New Perspectives in Algebraic Combinatorics (Berkeley, CA, 1996-1997), 91-147, Math. Sci. Res. Inst. Publ. 38, Cambridge Univ. Press, Cambridge, 1999.

[3] Barvinok, A.I. Computing the Ehrhart quasi-polynomial of a rational simplex. Manuscript 2005. Available at front.math.ucdavis.edu math.CO/0504444.

[4] de Klerk, E., Laurent, M., and Parrilo, P. A PTAS for the minimization of polynomials of fixed degree over the simplex. Manuscript 2004, available at http://homepages.cwi.nl/ monique/

[5] De Loera, J.A., Hemmecke, R., Tauzer, J. and Yoshida, R. Effective lattice point counting in rational convex polytopes. Journal of Symbolic Computation, vol 38, 4, 2004, 1273-1302.

[6] De Loera, J.A., Haws, D., Hemmecke, R., Huggins, P., Sturmfels, B., and Yoshida, R. Short rational functions for toric algebra and applications. Journal of Symbolic Computation, Vol. 38, 2, 2004, 959-973.

[7] Garey, M.R. and Johnson, D.S. Computers and Intractability: A Guide to the Theory of NP-Completeness. Freeman, San Francisco, 1979.

[8] Huggins, P.M. Lattice point enumeration via rational functions and applications to optimization and statistics. Senior undergraduate thesis, Department of mathematics, University of California, Davis, 2004.

[9] Khachiyan, L. and Porkolab, L. Integer optimization in convex semialgebraic sets. Discrete Comput. Geom. 23, 2000, 207-224.

[10] Jeroslow, R.G. There cannot be any algorithm for integer programming with quadratic constraints. Operations Research 21:1, 1973, 221-224.

[11] Jones, J.P. Universal Diophantine equation. J. of Symbolic Logic, 47 (3), 1982, 403-410.

[12] Laurent, M. A comparison of the Sherali-Adams, Lovász-Schrijver and Lasserre relaxations for 0-1 programming. Mathematics of Operations Research, 28, 3, 2003, 470-496.

[13] Lasserre, J.B. Global optimization with polynomials and the problem of moments. SIAM J. Optimization 11, 2001, 796-817.

[14] Lenstra, H.W. Jr. Integer Programming with a fixed number of variables. Mathematics of Operations Research, 8, 538-548

[15] Matiyasevich, Y. Hilbert's tenth problem. The MIT Press, Cambridge, London, 1993.

[16] Parrilo, P.A. and Sturmfels, B. Minimizing polynomial functions. In: Algorithmic and quantitative real algebraic geometry, DIMACS Series in Discrete Mathematics and Theoretical Computer Science, Vol. 60, pp. 83-99, AMS. 